\title{
Hämatologie und Onkologie in der Niederlassung - in Zeiten von COVID-19 eine Herausforderung
}

\author{
Interview mit Prof. Dr. Stephan Schmitz, Partner Medizinisches \\ Versorgungszentrum (MVZ) für Onkologie und Hämatologie, Praxis \\ am Sachsenring in Köln
}

Die ambulante Behandlung von onkologischen Patienten ist seit nahezu 20 Jahren eine wichtige Säule in der Krebsmedizin - auch in Zeiten von COVID-19. Prof. Dr. Stephan Schmitz ist nicht nur langjähriger Schriftleiter der best practice onkologie, er ist auch einer der Wegbereiter der ambulanten Krebsmedizin. Wir sprachen mit inm über Errungenschaften und aktuelle Herausforderungen in der hämatologischonkologischen Praxis.

best practice onkologie: Nach welchen Kriterien entscheiden Sie, ob Sie zurzeit Patienten direkt in Ihrer Praxis begegnen oder ob eine telefonische Betreuung ausreicht?

S. Schmitz: Hauptsächlich entscheiden wir das anhand der medizinischen Notwendigkeit. Alles weniger Dringende verschieben wir, und mittlerweile setzen wir auch Videosprechstunden ein. Häufig ist es allerdings absolut notwendig, Patienten zu sehen, um zum Beispiel die Nebenwirkungen der Krebstherapien zu überwachen und zu behandeln.

\section{》) Häufig ist es absolut notwendig, die Krebs- patienten zu sehen}

best practice onkologie: Welche Schutzmaßnahmen haben Sie konkret in Ihrer Praxis eingeführt, um eine Ansteckung mit SARS-CoV-2 zu verhindern?

S. Schmitz: Wir haben natürlich relativ schnell angefangen, die Praxisprozesse zu optimieren, um die Abstände zwischen den Patienten zu erhöhen. Wir haben die Terminkalender entzerrt und dafür unsere Arbeitszeiten verlängert, denn wir müssen die Patienten ja trotzdem weiter behandeln. In den Wartezonen sitzen die Patienten weiter auseinander, es befinden sich weniger Patienten in den Therapiebereichen, und es gibt Abstandsmarkierungen auf dem Boden. Außerdem haben wir Plexiglasscheiben als Spuckschutz und Mund-Nasen-Schutzmasken organisiert. Die Maßnahmen klappen gut, und die $\mathrm{Pa}$ tienten arbeiten auch gut mit.

best practice onkologie: Welche Schutzmaßnahmen empfehlen Sie Ihren Krebspatienten?

S. Schmitz: Den Krebspatienten empfehlen wir, genau wie alle anderen Menschen in Deutschland die Regeln des Robert Koch-Instituts konsequent zu befolgen. 
Hier steht eine Anzeige.

\section{Springer}


best practice onkologie: Welche Krebspatienten haben ein besonders hohes Risiko für eine Infektion?

S. Schmitz: Das sind entweder die Patienten, die eine sehr starke immunsuppressive Therapie erhalten, oder aber auch $\mathrm{Pa}$ tienten, die Grunderkrankungen haben, die schon mit einer Immunsuppression einhergehen.

best practice onkologie: Ist es für diese Patienten sinnvoll, die Krebsbehandlung zu verschieben?

S. Schmitz: Das muss man individuell bewerten, denn das hängt von der Krebserkrankung ab, von einer eventuell vorherrschenden immunsuppressiven Grunderkrankung sowie von anderen Komorbiditäten, von der Therapie, dem Therapieziel, den Laborparametern und vom Allgemeinzustand des Patienten. Alle diese Mosaiksteine ergeben ein Gesamtbild des Patienten. Davon ausgehend muss man entscheiden, ob die Therapie fortgeführt wird oder nicht. Man muss abwägen zwischen dem Therapieziel und dem Risiko des Patienten, sich mit SARS-CoV-2 zu infizieren. Ist die Therapie kurativ, wird man sie in der Regel fortführen wollen. Wir haben bis jetzt nur sehr wenige $\mathrm{Pa}$ tienten gehabt, wo eine Therapie aus COVID-19-Prophylaxe-Gründen verschoben werden musste.

\section{》) Man muss abwägen zwischen dem Therapie- ziel und dem Risiko des Patienten, sich mit SARS- CoV-2 zu infizieren}

best practice onkologie: Wie viele Ihrer Krebspatienten sind bereits mit SARSCoV-2 infiziert worden?

S. Schmitz: Bei uns in der Praxis ist mir kein Patient bekannt, der infiziert ist. Aber die Patienten werden ja auch nur getestet, wenn sie COVID-19-relevante Symptome haben, und es gibt genau wie in der Allgemeinbevölkerung keine Serientestungen.

best practice onkologie: Ihre Schwerpunktpraxis für Onkologie und Hämatologie haben Sie bereits vor 23 Jahren zusammen mit Dr. med. Hans Tilman Steinmetz

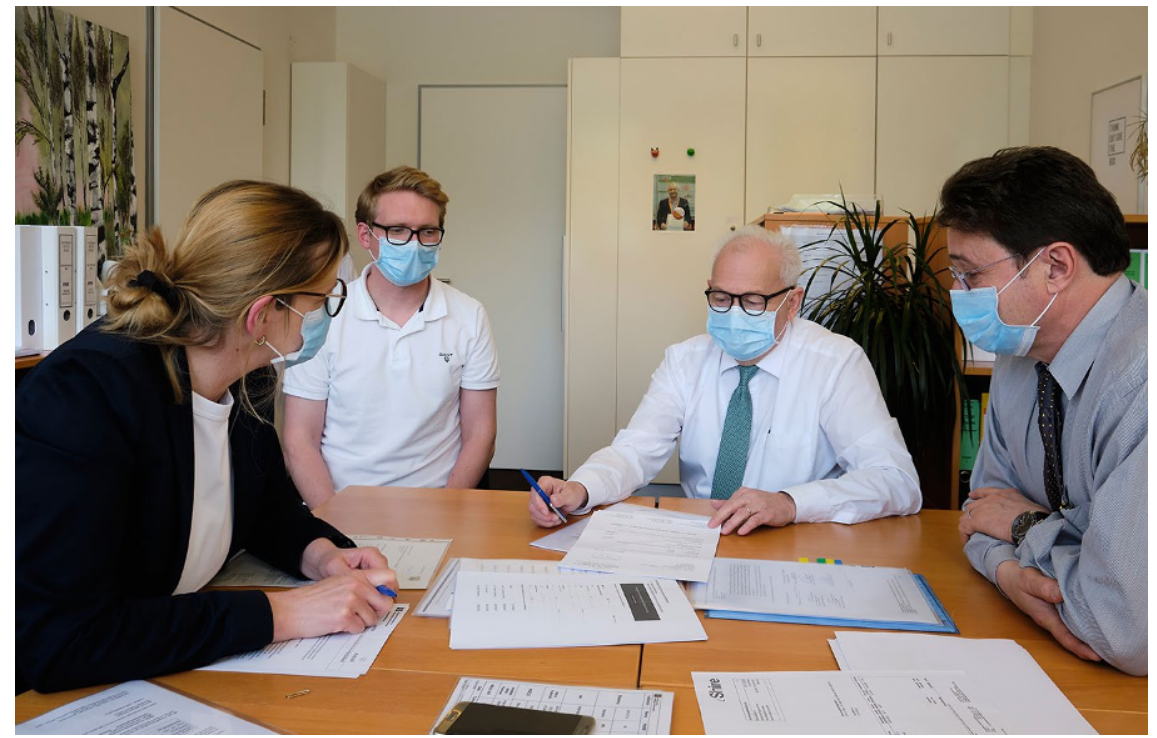

Bei der Mehrzahl der Patienten werden die Befunde in der wöchentlichen Besprechung der Studienabteilung und der internen Tumorkonferenz analysiert und die Behandlung abgestimmt

gegründet, wie kam es zu dieser Entscheidung?

S. Schmitz: Gute Frage! Wir haben uns jedenfalls beide sehr wohlgefühlt in der Uniklinik Köln. Wir waren beide Oberärzte, wir hatten beide lebenslange Arbeitsverträge. Es gab also keinen Druck, von dort weg zu gehen, aber die Selbstständigkeit hat uns gereizt. Wir wollten die ambulante Onkologie, die zu der Zeit noch in den Kinder- oder besser in den Jugendschuhen steckte, mitgestalten. In Köln gab es damals nur einen Kollegen, der als Onkologe niedergelassen war, sodass wir dachten, dass wir eine gute Chance hätten, uns zu entwickeln. Wir waren nicht naiv und haben vorher viele Marktrecherchen durchgeführt, und wir kannten natürlich auch die Patientenzahlen aus der Uniklinik. Aber wir hatten keine Vorstellung, wie sich das Gesundheitssystem nach 1997 verändern würde. Trotzdem haben wir es bisher nicht bereut, diesen Schritt gegangen zu sein.

\section{) Wir haben es bisher nicht bereut, diesen Schritt gegangen zu sein}

best practice onkologie: Sie haben in Ihrem $M V Z$ ein eigenes Studienzentrum auf- gebaut. Welche Studienerfolge haben Sie persönlich besonders gefreut?

S. Schmitz: In der Uniklinik Köln sind wir sozusagen mit den klinischen Studien zu Morbus Hodgkin sozialisiert worden. Diese Studien haben uns gezeigt, dass man durch gute klinische Studien tatsächlich einen relevanten Fortschritt für die Patienten generieren kann. An diesem Fortschritt wollten wir mitwirken. Gleichzeitig würden unsere Daten dann auch transparent der Studienzentrale vorliegen, was zu einer Qualitätssicherung führt. Fortschritt und Qualitätssicherung waren die beiden Aspekte, warum wir eine Studienabteilung aufgebaut haben. Wir waren also von Anfang an überzeugt, dass wir an Studien teilnehmen wollen, insbesondere an den so genannten „investigator-initiated studies“. So ein Studienprogramm aufzubauen, ist nicht einfach, das muss auch finanziert werden. Das war harte Arbeit. Wir haben uns auch an internationalen Zulassungsstudien beteiligt.

Neben den ,investigator-initiated studies“ haben sich dann die Registerstudien entwickelt. Die waren vor 20 Jahren nicht wirklich wissenschaftlich anerkannt. Mittlerweile ist das komplett anders. Heute weiß man, dass Registerstudien hoch relevant sind für die Versorgungsforschung. Hier haben wir uns auch engagiert, wir haben eigene Register aufgebaut. Dr. Steinmetz hat sich mit dem 


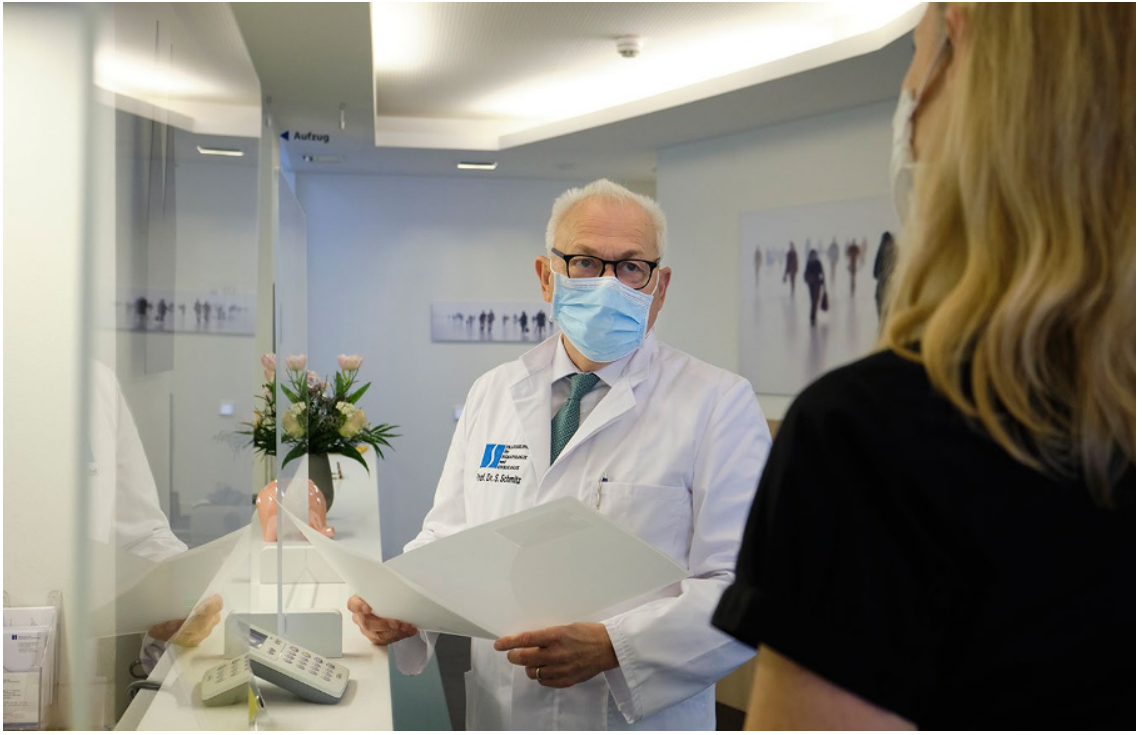

Für ein kurzes Gespräch im Wartebereich ist auch im Praxisalltag meist Zeit

Thema Anämie unter Chemotherapie ausführlich beschäftigt und große Studien gemacht. Und wir haben ein Register zum myelodysplastischen Syndrom mit über 100 teilnehmenden Praxen aufgebaut, in das knapp 2500 Patienten eingeschlossen worden sind. Wir sind überzeugt, dass Registerstudien die Versorgung verbessern können.

\section{》) Es ist entscheidend, dass man sich um seine Patienten kümmert und sich für sie verantwortlich fühlt}

best practice onkologie: Von Anfang an war Ihnen das Qualitätsmanagement (QM) Ihrer Praxis wichtig. Als zweite onkologische Praxis in Deutschland hat Ihr QM-System das Qualitätssiegel 1998 durch den TÜV-CERT Rheinland erhalten. Was macht für Sie eine qualitativ hochwertige Krebsbehandlung aus?

S. Schmitz: Auf jeden Fall nicht allein, dass man ein Zertifikat hat. Eine gute onkologische Behandlung entwickelt sich meines Erachtens aus einer Kombination aus externer und interner Evidenz. Der Onkologe muss natürlich die wissenschaftlichen Daten kennen, das meine ich mit externer Evidenz. Aber

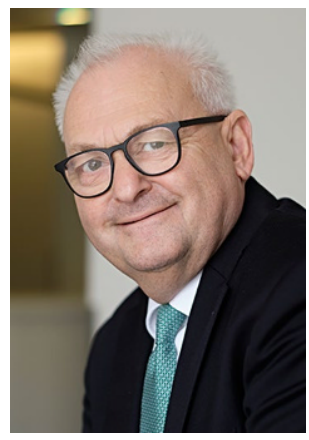

\section{Zur Person} practice onkologie. des Onkologen und die Unabhängigkeit der Therapieentscheidung sind extrem wichtig. Am Ende des Tages ist es entscheidend - und das kann man nicht messen -, dass man sich um seine $\mathrm{Pa}$ tienten kümmert. Das wird häufig unterschätzt. Kümmern heißt, dass man sich für den Patienten verantwortlich fühlt, dass man Dingen, die unklar sind, hinterhergeht, dass man Originalbefunde an Land zieht, dass man für den Patienten ein Gesamtkonzept entwickelt. Ich kann zum Beispiel den Satz schwer ertragen: „Die Tumorkonferenz hat entschieden“. Eine Tumorkonferenz ist ein sehr wichtiges Beratungsgremium, aber sie kann keine Patienten behandeln, das müssen die Onkologen selbst tun. Und deswegen liegt die letztendliche Entscheidung immer beim Onkologen und dem individuellen Patienten. Das soll die Tumorkonferenz in keiner Weise reduzieren, aber es muss klar sein, wer sich verantwortlich fühlt. Das müssen wir auch unseren jungen Kollegen vermitteln. Denn das macht

Prof. Dr. Stephan Schmitz ist Facharzt für Innere Medizin, Hämatologie und Onkologie und Arzt für Palliativmedizin. Zunächst erlangte der heute vierfache Vater ein Diplom in Physik, anschließend entschied er sich mit 27 Jahren für das Studium der Medizin in Aachen und in seiner Heimatstadt Köln. An seine Approbation als Arzt und seine Promotion 1988 schloss er seine Facharztausbildung an. Von 1994 bis 1996 war er Oberarzt der Klinik I für Innere Medizin der Universität zu Köln. Gemeinsam mit seinem Kollegen Dr. med. Hans Tilman Steinmetz gründete er 1997 die Gemeinschaftspraxis für Hämatologie und Onkologie am Sachsenring in Köln. Die beiden Ärzte bauten in ihrem MVZ ein eigenes Studienzentrum auf, mit dem sie sich bis heute an nationalen und internationalen Studien beteiligen und selbst eigene Studien und Registerstudien durchführen. Fortwährend hat sich Schmitz für die Stärkung der ambulanten Onkologie eingesetzt. So übernahm er im Jahr 2000 den Vorsitz des Berufsverbands der Niedergelassenen Hämatologen und Onkologen in Deutschland (BNHO) und führte diesen über 18 Jahre lang. In dieser Zeit erstritt er u. a. mit dem BNHO die Einführung der ambulanten spezialärztlichen Versorgung (ASV), sein MVZ stellte daraufhin 2018 das erste ASV-Team für Tumorerkrankungen des Bauchraums in Köln zusammen mit dem Krankenhaus der Augustinerinnen und dem Antonius-Hospital auf. Schmitz möchte durch interdisziplinäre Kooperationen die bestmögliche Behandlung für seine Patienten erreichen. Deshalb gründete er mit seinen Kollegen die in Deutschland einmalige Ambulante Fachübergreifende Onkologie. Zudem wurde auf Initiative seines MVZ das Darmzentrum Köln gegründet. Weiter ist Schmitz Mitbegründer und onkologischer Sprecher des Deutschen Onkologie Netzes (DON). Seit fast 15 Jahren ist Schmitz bereits Herausgeber und Schriftleiter der Zeitschrift best 


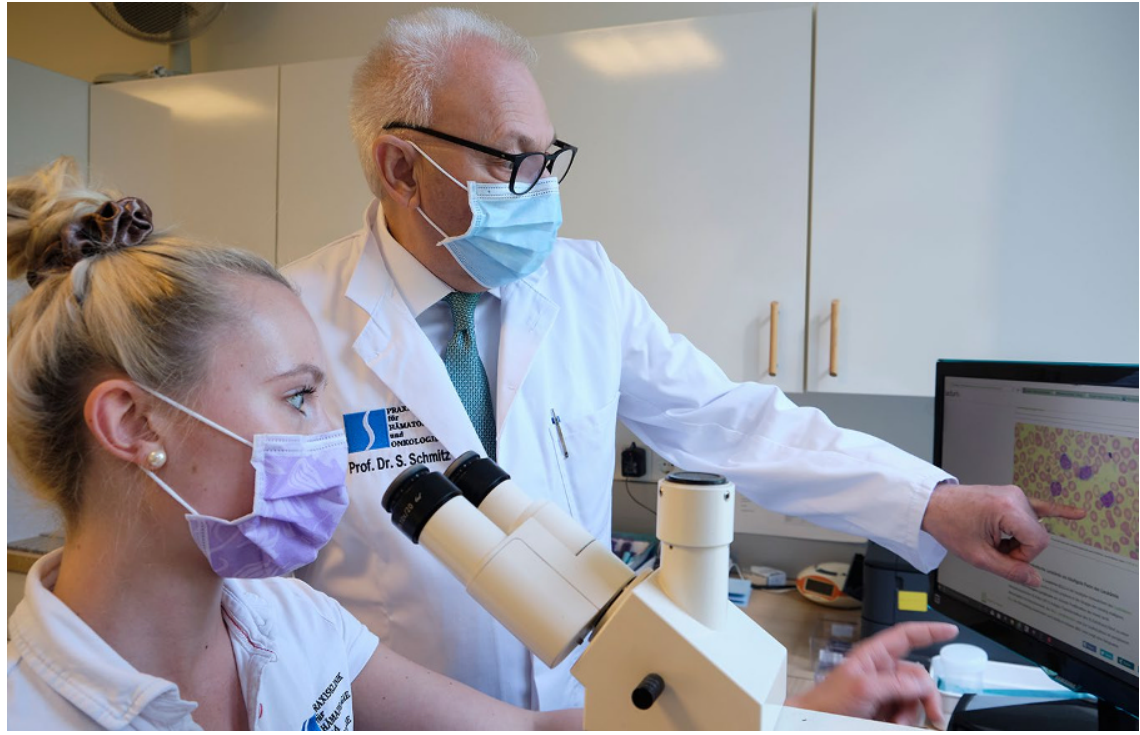

Ein gutes Mikroskop und analysesichere Mitarbeiter sind bei der Diagnostik hämatologischer Erkrankungen nach wie vor unverzichtbar

am Ende den Unterschied aus zwischen einer vielleicht mittelmäßigen und einer guten onkologischen Betreuung.

Sich um den Patienten kümmern bedeutet auch, dass man sich innerhalb eines Netzwerks organisieren sollte. Denn Onkologie ist ganz klar multidisziplinär, interdisziplinär und intersektoral, und man kann meines Erachtens nur einen Patienten gut durch den Dschungel einer Krebserkrankung führen, indem man diese Netzwerke kennt und sie kompetent nutzen kann.

\section{I) Man kann einen Patien- ten nur gut durch den Dschungel einer Krebser- krankung führen, indem man Netzwerke kennt und diese kompetent nutzen kann}

best practice onkologie: Ihre Praxis hat über die Jahre enge Kooperationen geknüpft und u. a. die in Deutschland einmalige „Ambulante Fachübergreifende Onkologie“ (AFO) gegründet. Was verbirgt sich dahinter?

S. Schmitz: Wie gesagt, sind wir davon überzeugt, dass die interdisziplinä- re Versorgung für die Patienten am besten ist. Das ist der Grund, warum wir in Köln mit sechs niedergelassenen Urologen, die auch onkologische Patienten betreuen, eine Praxisgemeinschaft gegründet haben. Die Urologen behandeln jetzt die onkologischen Patienten in unserer Infrastruktur. Wir glauben, dass das ein Modell ist, mit dem man sehr gut zusammenarbeiten kann. Berufspolitische Grabenkämpfe mit den so genannten Organonkologen sind auf Dauer nicht sinnvoll. Ich glaube, man sollte das Beste aus beiden Welten zusammenführen.

best practice onkologie: Auch das Deutsche Onkologie Netz (DON) haben Sie und Ihre MVZ-Kollegen mitbegründet. Wie funktioniert dieses Netzwerk?

S. Schmitz: Das DON ist ein Netzwerk aus onkologischen, arztgeführten MVZ. Wir wollen damit die wohnortnahe ambulante Versorgung von Krebspatienten verbessern. In solchen Netzwerkstrukturen kann man viele Dinge gemeinsam organisieren und entwickeln, die man alleine nicht gut machen kann. Dazu gehört unter anderem, die EDV-Infrastruktur weiterzuentwickeln, eine gemeinsame Qualitätssicherung $\mathrm{zu}$ etablieren und gemeinsam gegenüber den Patienten aufzutreten. Es verändert sich unheimlich viel in unserem Gesundheitssystem, auch für die ambulanten Ärzte. Wir versuchen in unserem Netzwerk, Angebote zu entwickeln, die diese Veränderungen aufnehmen. best practice onkologie: Sie waren lange Vorsitzender des Berufsverbands der Niedergelassenen Hämatologen und Onkologen in Deutschland (BNHO) und konnten u. a. die ambulante spezialfachärztliche Versorgung (ASV) mit auf den Weg bringen. Ist die ASV in der Versorgung angekommen?

S. Schmitz: Die ASV war tatsächlich ein Thema, das wir vom Berufsverband jahrelang propagiert haben. Das war ein sehr langer Weg. Die ersten Gespräche haben wir noch mit Ulla Schmidt im Jahr 2004 und später mit Philipp Rösler geführt. Am Ende hat dann Daniel Bahr die Neuregelung des $₫ 116$ b SGB V im Jahr 2012 erwirkt. Die alte Version hatte die Krankenhäuser nach unserer Meinung privilegiert und ihnen einen massiven Wettbewerbsvorteil verschafft. Wir wollten einen dritten Sektor haben, in dem Krankenhäuser und niedergelassene Ärzte zu gleichen Spielregeln onkologische Patienten versorgen können. Darüber hinaus sollten die Krankenkassen und nicht die Ärzte das Morbiditätsrisiko für die Behandlung der Krebspatienten übernehmen. Beide Ziele sind im Prinzip mit der ASV gesetzlich verankert worden. Allerdings ist die Umsetzung der ASV in das praktische Leben bisher nicht wirklich gelungen. Das hat administrative, aber auch andere Gründe. Die Idee war gut, die Umsetzung ist noch verbesserungswürdig.

best practice onkologie: Gerade in der Krise fallen Versorgungslücken besonders auf. Welche Schritte sind weiter nötig, um die ambulante Onkologie und Hämatologie $z u$ verbessern?

S. Schmitz: Natürlich gibt es immer Versorgungslücken, aber gerade jetzt in der COVID-19-Situation haben wir bewiesen, dass das deutsche Gesundheitssystem extrem leistungsfähig ist. Das liegt daran, weil es nicht zentral, sondern dezentral bzw. föderalistisch organisiert ist. Es gibt Universitätskliniken, Krankenhäuser und niedergelassene Fachärzte. Diesen Dreiklang müssen wir noch breiter vernetzen und sozusagen besser orchestrieren. Wir müssen den Innovationsschub, den wir in der Onkologie zum Glück haben, und den Innovationstransfer gemeinsam gut organisieren. Mein Grundsatz ist, dass dies weiterhin dezentral passieren sollte. Wir sollten das Know-how in die Fläche bringen zu den Krankenhäusern und in die Praxen. Um- 


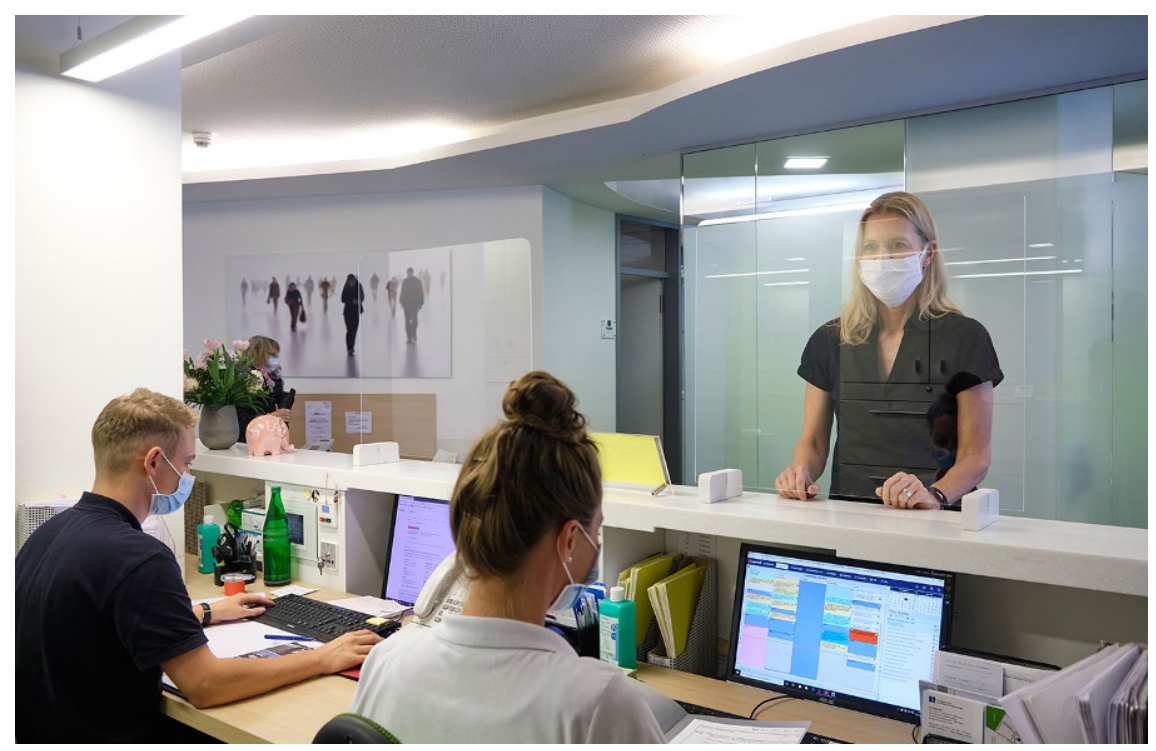

Auch in Zeiten von Corona sorgt ein gutes Praxisteam nicht nur für reibungslose Abläufe, es gibt auch den Patienten ein Gefühl von Sicherheit und Geborgenheit

gekehrt müssen wir dann aber auch Informationen aus der Versorgung wieder in die forschenden Institutionen zurückspielen.

best practice onkologie: Was würden Sie jungen Kollegen empfehlen, die sich heutzutage als Onkologe und Hämatologe niederlassen wollen?

S. Schmitz: Erst einmal, dass sie keine Angst haben müssen, diesen Schritt zu gehen. Viele junge Ärzte sind, glaube ich, sehr verunsichert, weil Medien oder ganz bestimmte Interessengruppen propagieren, dass die wirtschaftliche Basis für die Niederlassung nicht mehr gegeben ist oder dass Regresse drohen. Das sind alles Dinge, die man berücksichtigen muss, aber die sollten nicht dazu führen, dass man sich nicht als Hämatologe und Onkologe niederlässt. Natürlich müssen die jungen Kollegen engagiert sein, aber das sind sie in der Regel. Sie müssen sich möglicherweise überlegen, ob sie alleine, in kleinen Strukturen oder in Netzwerken wie zum Beispiel dem Deutschen Onkologie Netz arbeiten wollen. Als selbstständiger Arzt zu arbeiten, ist immer noch eine hochinteressante Tätigkeit. Man kann seine Vorstellung von seiner Arbeitswelt und davon, wie man Patienten behandelt, in der Niederlassung sehr viel besser umsetzen als in angestellten Situationen. Denn man kann selbst entscheiden, wie man sein Arbeitsumfeld organisiert und strukturiert. Das ist ein großes Gut. best practice onkologie: Herr Professor Schmitz, vielen Dank für das interessante Gespräch.

Das Interview führte: Sabrina Kempe, Dresden

Fotos: Heiko Specht

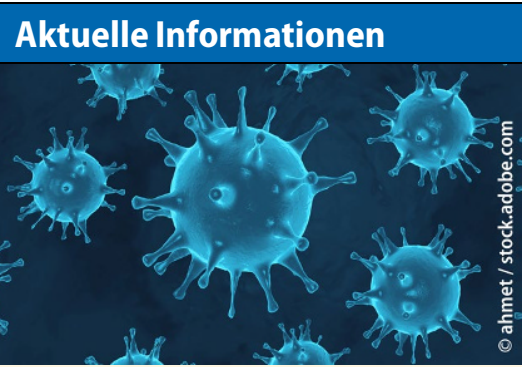

\section{Aktuelle Entwicklung zu COVID-19 bei Springer Nature und Springer Medizin}

Springer Nature und Springer Medizin unterstützen die globale Reaktion auf die COVID-19-Pandemie, indem ein schneller und direkter $\mathbf{Z u}$ gang zu den neuesten verfügbaren Forschungsergebnissen und Daten ermöglicht wird.

Auf der Homepage SpringerMedizin.de finden Sie ein immer aktuelles Dossier mit Beiträgen, Forschungsarbeiten und Ergebnissen zu SARS-CoV-2 sowie relevanten Links.

Darin z.B. auch die kürzlich publizierte Empfehlung von DIVI, DGIIN, DGAI und DGP zur Intensivtherapie von Patienten mit COVID-19.

Springer Nature arbeitet mit globalen Organisationen zusammen, und verlinkt über SpringerNature.com/de auf eine eigene Landingpage mit einer Vielzahl an Information sowie freiem Zugriff auf die COVID-19-Contentplattformen von Nature Research, BioMed Central (BMC) und Springer.

Das Dossier zu Coronavirus / Covid-19 von Springer Medizin finden Sie hier: www.springermedizin.de/covid-19

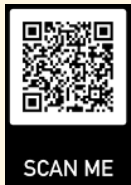

